\title{
Uniqueness and zeros of q-shift difference polynomials sharing one value
}

\author{
HARINA P. WAGHAMORE ${ }^{\dagger}$ AND SANGEETHA ANAND
}

\author{
Date of Receiving : $\quad 16.05 .2017$ \\ Date of Revision : 18.08 .2017 \\ Date of Acceptance : 24.08 .2017
}

\begin{abstract}
In this paper, we study uniqueness problems and zeros of q-shift difference polynomials of entire and meromorphic functions with zero order in the complex plane. The results of this paper extends the results obtained by Q. Zhao and J. Zhang [9].
\end{abstract}

\section{Introduction and main results}

The fundamental theorems and the standard notations of the Nevanlinna value distribution theory will be used in this article. By a meromorphic function we always mean a non-constant analytic function in the whole complex plane except at possible poles. If no poles occur, then it reduces to an entire function (see $[1,2,3])$.

We use $S(r, f)$ to denote any quantity satisfying $S(r, f)=o\{T(r, f)\}$, as $r \longrightarrow+\infty$, possibly outside a set of logarithmic density zero. Let $f(z)$ and $g(z)$ be two non-constant meromorphic functions in the complex plane, $a \in \mathbb{C} \cup\{\infty\}$, we say that $f$ and $g$ share the value $a$ IM (ignoring multiplicities) if $f-a$ and $g-a$ have the same zeros, they share the value $a$ CM (counting multiplicities) if $f-a$ and $g-a$ have the same zeros with the same multiplicities. When $a=\infty$, the zeros of $f-a$ means the poles of $f$.

Let $p$ be a positive integer and $a$ be a complex constant, then we denote by $N_{p}\left(r, \frac{1}{f-a}\right)$ the counting function of the zeros of $f-a$, where an $\mathrm{m}$-fold zero is counted $\mathrm{m}$ times if $m \leq p$ and $p$ times if $m>p$.

In 1959, Hayman [4] proved that $f^{n} f^{\prime}$ takes every non-zero complex value infinitely often if $n \geq 3$. Yang and Hua [5] obtained some results about the uniqueness problems for entire functions. Since then the difference has become a subject of great interest (see $[5,6,7,8,14])$.

2010 Mathematics Subject Classification. 30D35.

Key words and phrases. Uniqueness, Entire functions, Meromorphic functions, Difference polynomials, zeros, q-shift.

Communicated by: Sanjib Dutta

${ }^{\dagger}$ Corresponding author. 